\title{
Relation between catalyst-assisted transformation and multiple-copy transformation for bipartite pure states
}

\author{
Yuan Feng,, , 因 Runyao Duan,, 团 and Mingsheng Ying1, 团 \\ ${ }^{1}$ State Key Laboratory of Intelligent Technology and Systems, \\ Department of Computer Science and Technology Tsinghua University, Beijing, China, 100084
}

(Dated: November 11, 2018)

\begin{abstract}
We show that in some cases, catalyst-assisted entanglement transformation cannot be implemented by multiple-copy transformation for pure states. This fact, together with the result we obtained in [R. Y. Duan, Y. Feng, X. Li, and M. S. Ying, Phys. Rev. A 71, 042319 (2005)] that the latter can be completely implemented by the former, indicates that catalyst-assisted transformation is strictly more powerful than multiple-copy transformation. For purely probabilistic setting we find, however, these two kinds of transformations are geometrically equivalent in the sense that the sets of pure states which can be converted into a given pure state with maximal probabilities not less than a given value have the same closure, no matter catalyst-assisted transformation or multiple-copy transformation is used.
\end{abstract}

PACS numbers: 03.67.Mn,03.65.Ud

\section{INTRODUCTION}

Quantum entanglement, which is essential in quantum information processing such as quantum cryptography [1], quantum superdense coding [2] and quantum teleportation [3], has been extensively studied. One fruitful research direction on quantum entanglement is to discuss the possibility of transforming a bipartite entangled pure state into another one allowing only local operations on the separate subsystems respectively and classical communication between them (or LOCC for short). The asymptotic case when arbitrarily large number of copies are provided is considered by Bennett and his collaborators [4]. While in deterministic and finite manner, the first and significant step was made by Nielsen 5 ] who discovered the connection between the theory of majorization in linear algebra [6] and entanglement transformation. Nielsen proved that a bipartite entangled pure state $\left|\psi_{1}\right\rangle$ can be transformed into another bipartite entangled pure state $\left|\psi_{2}\right\rangle$ by LOCC if and only if $\lambda_{\psi_{1}} \prec \lambda_{\psi_{2}}$, where the probability vectors $\lambda_{\psi_{1}}$ and $\lambda_{\psi_{2}}$ denote the Schmidt coefficient vectors of $\left|\psi_{1}\right\rangle$ and $\left|\psi_{2}\right\rangle$, respectively. Here the symbol $\prec$ stands for 'majorization relation'. Generally, an $n$-dimensional real vector $x$ is said to be majorized by another $n$-dimensional real vector $y$, denoted by $x \prec y$, if the following relations hold:

$$
\sum_{i=1}^{l} x_{i}^{\downarrow} \leq \sum_{i=1}^{l} y_{i}^{\downarrow} \quad \text { for any } \quad 1 \leq l \leq n,
$$

with equality holding when $l=n$, where $x^{\downarrow}$ denotes the vector obtained by rearranging the components of $x$ in nonincreasing order.

\footnotetext{
*Electronic address: feng-y@tsinghua.edu.cn

$\dagger$ Electronic address: dry02@mails.tsinghua.edu.cn

‡Electronic address: yingmsh@tsinghua.edu.cn
}

Nielsen's theorem gives a necessary and sufficient condition when two entangled pure states are comparable in the sense that one can be transformed into another by LOCC. There exist, however, incomparable states such that any one cannot be transformed into another only using LOCC. To treat the case of transformations between incomparable states, Vidal 7] generalized Nielsen's work by allowing probabilistic transformations. He found that the maximal probability of transforming $\left|\psi_{1}\right\rangle$ into $\left|\psi_{2}\right\rangle$ by LOCC can be calculated by

$$
P\left(\left|\psi_{1}\right\rangle \rightarrow\left|\psi_{2}\right\rangle\right)=\min _{1 \leq l \leq n} \frac{E_{l}\left(\lambda_{\psi_{1}}\right)}{E_{l}\left(\lambda_{\psi_{2}}\right)}
$$

where $E_{l}(x)$ denotes $\sum_{i=l}^{n} x_{i}^{\downarrow}$.

In Ref. [8], Jonathan and Plenio discovered a very surprising phenomenon that sometimes an entangled state can enable otherwise impossible entanglement transformations without being consumed at all. A simple but well known example is $\left|\psi_{1}\right\rangle \nrightarrow\left|\psi_{2}\right\rangle$ but $\left|\psi_{1}\right\rangle \otimes|\phi\rangle \rightarrow$ $\left|\psi_{2}\right\rangle \otimes|\phi\rangle$, where $\left|\psi_{1}\right\rangle=\sqrt{0.4}|00\rangle+\sqrt{0.4}|11\rangle+\sqrt{0.1}|22\rangle+$ $\sqrt{0.1}|33\rangle,\left|\psi_{2}\right\rangle=\sqrt{0.5}|00\rangle+\sqrt{0.25}|11\rangle+\sqrt{0.25}|22\rangle$, and $|\phi\rangle=\sqrt{0.6}|44\rangle+\sqrt{0.4}|55\rangle$. The role of the state $|\phi\rangle$ is just like a catalyst in a chemical process. Daftuar and Klimesh [9] examined catalyst-assisted entanglement transformation and derived some interesting results. In [10], we investigated catalyst-assisted transformation in probabilistic setting. A necessary and sufficient condition was presented under which there exist partial catalysts that can increase the maximal transforming probability of a given entanglement transformation. The mathematical structure of catalyst-assisted probabilistic transformation was also carefully investigated.

Another interesting phenomenon of entanglement transformation was noticed by Bandyopadhyay et al. [1]. In some occasions, increasing the number of copies of the original state can also help entanglement transformations. Take the above example. Instead of introducing a catalyst state $|\phi\rangle$, providing 3 copies of $\left|\psi_{1}\right\rangle$ is 
also sufficient to transform these copies together into the same number of $\left|\psi_{2}\right\rangle$. A question naturally arises here is: what is the relation between catalyst-assisted entanglement transformation and multiple-copy transformation? In [12], we found that multiple-copy entanglement transformation can be completely implemented by catalystassisted one. Furthermore, the mixing of these two has also the same power as pure catalyst-assisted transformation. In other words, any transformation which can be realized collectively on multiple copies and with the aid of a catalyst can be exactly implemented by only providing some appropriate catalyst. Later on, we proved that these two kinds of transformations are asymptotically equivalent in the sense that they can simulate each other's ability to implement a desired transformation with the same optimal success probability, when the dimension of catalysts and the number of copies provided tend to infinity [13].

The contribution of the current paper is twofold. First, we show that in some cases catalyst-assisted entanglement transformation is strictly more powerful than multiple-copy one by deriving a sufficient condition when the former cannot be implemented by the latter. Second, for purely probabilistic setting we find, however, these two kinds of transformations are geometrically equivalent. That is, no matter catalyst-assisted transformations or multiple-copy transformations are used, the sets of quantum states that can be converted into a given state with maximal probabilities not less than a given value have the same closure. It is worth noting that the geometrical equivalence between these two kinds of transformations proved in the current paper is different from the asymptotical equivalence shown in 13. We will elaborate the difference at the end of Section III after necessary notations have been introduced.

For simplicity, in what follows we denote a bipartite pure state by the probability vector of its Schmidt coefficients. This will not cause any confusion because it is well known that the fundamental properties of a bipartite pure state under LOCC are completely determined by its Schmidt coefficients. Therefore, from now on, we consider only probability vectors (sometimes we even omit the normalization of a nonnegative vector to be a probability one) instead of quantum states and always identify a probability vector with the bipartite pure state represented by it.

\section{DETERMINISTIC CASE}

In this section, we study the relation between catalystassisted transformation and multiple-copy transformation in deterministic case. First, we introduce some notations.

Denote by $V^{n}$ the set of all $n$-dimensional nonnegative vectors and let $x, y, \cdots$ range over $V^{n}$. Let

$$
S(y)=\left\{x \in V^{n} \mid x \prec y\right\}
$$

be the set of states that can be transformed into $y$ by LOCC directly,

$T(y)=\left\{x \in V^{n} \mid \exists\right.$ probability vector $\left.c, x \otimes c \prec y \otimes c\right\}$

be the set of states that can be transformed into $y$ by LOCC with the aid of some catalyst, and

$$
M(y)=\left\{x \in V^{n} \mid \exists \text { integer } k \geq 1, x^{\otimes k} \prec y^{\otimes k}\right\}
$$

the set of states which, when some appropriate number of copies are provided, can be transformed into the same number of $y$ by LOCC.

Lemma 1 Suppose $x \in T(y)$ and $x^{\prime} \in T\left(y^{\prime}\right)$. Then $\bar{x} \in$ $T(\bar{y})$ where $\bar{x}=x \oplus x^{\prime}$ and $\bar{y}=y \oplus y^{\prime}$.

Proof. By definition, $x \in T(y)$ and $x^{\prime} \in T\left(y^{\prime}\right)$ imply that there exist $c$ and $c^{\prime}$ such that $x \otimes c \prec y \otimes c$ and $x^{\prime} \otimes c^{\prime} \prec y^{\prime} \otimes c^{\prime}$. It can be easily checked that the vector $c \otimes c^{\prime}$ serves as a catalyst for the transformation from $\bar{x}$ to $\bar{y}$, that is, $\bar{x} \otimes c \otimes c^{\prime} \prec \bar{y} \otimes c \otimes c^{\prime}$. Thus $\bar{x} \in T(\bar{y})$.

The following lemma, important in its own right, is a powerful tool which gives us a sufficient condition on $x$ and $y$ such that they are incomparable in any multiplecopy transformations. In other words, any number of $x$ cannot be collectively transformed into the same number of $y$ using LOCC.

Lemma 2 Suppose $x$ and $y$ are two nonincreasingly arranged $n$-dimensional probability vectors, $x_{1}=y_{1}$ but $x \nprec y$. Let

$$
d=\min \left\{l: 1 \leq l \leq n, \sum_{i=1}^{l} x_{i}>\sum_{i=1}^{l} y_{i}\right\} .
$$

Denote by $t_{1}$ the number of components in $x$ which are equal to $x_{1}$, while $t_{2}$ the number of components in $y$ which are equal to $y_{1}$. If $t_{1}=t_{2}=t$ and

$$
x_{1} x_{d} \geq x_{t+1}^{2} \quad \text { and } \quad y_{1} y_{d} \geq y_{t+1}^{2},
$$

then $x \notin M(y)$.

Proof. First, it is obvious that $1 \leq t<d<n$. From the assumption Eq.(7), we have for any integer $k \geq 1$, the components of $x^{\otimes k}$ and $y^{\otimes k}$ can be arranged nonincreasingly as follows

$$
\begin{aligned}
\left(x^{\otimes k}\right)^{\downarrow}= & x_{1}^{k} \oplus\left(x_{1}^{k-1} x_{2}\right)^{\oplus k} \oplus \cdots \oplus\left(x_{t}^{k-1} x_{t-1}\right)^{\oplus k} \oplus x_{t}^{k} \\
& \oplus\left(x_{1}^{k-1} x_{t+1}\right)^{\oplus k} \oplus \cdots \oplus\left(x_{t}^{k-1} x_{t+1}\right)^{\oplus k} \oplus \cdots \\
& \oplus\left(x_{1}^{k-1} x_{d}\right)^{\oplus k} \oplus \cdots \oplus\left(x_{t}^{k-1} x_{d}\right)^{\oplus k} \oplus \cdots
\end{aligned}
$$

and

$$
\begin{aligned}
\left(y^{\otimes k}\right)^{\downarrow}= & y_{1}^{k} \oplus\left(y_{1}^{k-1} y_{2}\right)^{\oplus k} \oplus \cdots \oplus\left(y_{t}^{k-1} y_{t-1}\right)^{\oplus k} \oplus y_{t}^{k} \\
& \oplus\left(y_{1}^{k-1} y_{t+1}\right)^{\oplus k} \oplus \cdots \oplus\left(y_{t}^{k-1} y_{t+1}\right)^{\oplus k} \oplus \cdots \\
& \oplus\left(y_{1}^{k-1} y_{d}\right)^{\oplus k} \oplus \cdots \oplus\left(y_{t}^{k-1} y_{d}\right)^{\oplus k} \oplus \cdots
\end{aligned}
$$


where by $\alpha^{\oplus k}$ we denote the vector

$$
\underbrace{(\alpha, \alpha, \cdots, \alpha)}_{k \text { times }} \text {. }
$$

Here we only write out explicitly the largest $t^{k}+k t(d-t)$ components of $x^{\otimes k}$ and $y^{\otimes k}$ since it is enough for our argument. Notice that $x_{1}=\cdots=x_{t}>x_{t+1}$ and $y_{1}=$ $\cdots=y_{t}>y_{t+1}$. It is now easy to check that $x^{\otimes k} \nprec y^{\otimes k}$ since when taking $l=t^{k}+k t(d-t)$, we have

$$
\begin{aligned}
\sum_{i=1}^{l}\left(x^{\otimes k}\right)_{i}^{\downarrow} & =\left(t x_{1}\right)^{k}+k t x_{1}^{k-1} \sum_{i=t+1}^{d} x_{i} \\
& >\left(t y_{1}\right)^{k}+k t y_{1}^{k-1} \sum_{i=t+1}^{d} y_{i} \\
& =\sum_{i=1}^{l}\left(y^{\otimes k}\right)_{i}^{\downarrow} .
\end{aligned}
$$

So $x \notin M(y)$ by the arbitrariness of $k$.

If we take $x$ and $y$ as in Lemma 2 but $x_{n}=y_{n}$ instead of $x_{1}=y_{1}$. Let

$$
d=\max \left\{l: 1 \leq l \leq n, \sum_{i=l}^{n} x_{i}<\sum_{i=l}^{n} y_{i}\right\} .
$$

Denote by $t_{1}$ the number of components in $x$ which are equal to $x_{n}$ while $t_{2}$ the number of components in $y$ which are equal to $y_{n}$. If $t_{1}=t_{2}=t, x_{n} x_{d} \leq x_{n-t}^{2}$, and $y_{n} y_{d} \leq y_{n-t}^{2}$, then we can also deduce that $x \notin M(y)$.

Using the lemmas above, we can now prove that $T(y) \neq M(y)$ for some probability vector $y$ by deriving a sufficient condition under which $T(y) \nsubseteq M(y)$, as the following theorem states.

Theorem 3 Suppose $y$ is a nonincreasingly arranged $n$ dimensional probability vector. Denote by $t$ and $m$ the numbers of components which are equal to $y_{1}$ and which are equal to $y_{n}$, respectively. Let $d$ be the minimal index of the components which are less than $y_{t+1}$. That is,

$$
y_{1}=\cdots=y_{t}>y_{t+1}=\cdots=y_{d-1}>y_{d},
$$

and

$$
y_{n-m}>y_{n-m+1}=\cdots=y_{n}
$$

If $d<n-m$ and $y_{1} y_{d} \geq y_{t+1}^{2}$, then $T(y) \nsubseteq M(y)$.

Proof. Take a positive number $\epsilon$ such that

$\epsilon<\min \left\{\frac{d-t-1}{d-t}\left(y_{d-1}-y_{d}\right), \frac{m}{m+1}\left(y_{n-m}-y_{n-m+1}\right)\right\}$.
Define two $(n-t)$-dimensional nonnegative vectors $\bar{x}$ and $\bar{y}$ as follows

$$
\begin{aligned}
\bar{x}= & \left(y_{t+1}-\frac{\epsilon}{\triangle}, \cdots, y_{d-1}-\frac{\epsilon}{\triangle}, y_{d}+\epsilon, y_{d+1}, \cdots,\right. \\
& \left.y_{n-m-1}, y_{n-m}-\epsilon, y_{n-m+1}+\frac{\epsilon}{m}, \cdots, y_{n}+\frac{\epsilon}{m}\right)
\end{aligned}
$$

and

$$
\bar{y}=\left(y_{t+1}, y_{t+2}, \cdots, y_{n}\right) .
$$

Here $\triangle=d-t-1$. It is easy to check that $\bar{x}$ and $\bar{y}$ are both nonincreasingly arranged, and $\bar{x} \prec \bar{y}$. Furthermore, $\bar{x}$ is in the interior of $T(\bar{y})$ by Lemma 1 in $[9]$ since $\bar{x}_{1}<\bar{y}_{1}$ and $\bar{x}_{n-t}>\bar{y}_{n-t}$. So there exists a sufficiently small but positive $\delta$ such that $\bar{x}^{\prime} \in T(\bar{y})$ where

$$
\begin{aligned}
& \bar{x}^{\prime}=\left(y_{t+1}-\frac{\epsilon}{\triangle}, \cdots, y_{d-1}-\frac{\epsilon}{\triangle}, y_{d}+\epsilon+\delta, y_{d+1}, \cdots,\right. \\
& \left.y_{n-m-1}, y_{n-m}-\epsilon-\delta, y_{n-m+1}+\frac{\epsilon}{m}, \cdots, y_{n}+\frac{\epsilon}{m}\right) .
\end{aligned}
$$

Now define $x$ as the direct sum of the vectors $\left(y_{1}, \ldots, y_{t}\right)$ and $\bar{x}^{\downarrow}$, that is

$$
x=\left(y_{1}, \cdots, y_{t}, \bar{x}^{\downarrow}\right) .
$$

By Lemma 1 we have $x \in T(y)$. On the other hand,

$$
\begin{gathered}
\sum_{i=1}^{d} x_{i}=\sum_{i=1}^{d} y_{i}+\delta>\sum_{i=1}^{d} y_{i}, \\
\sum_{i=1}^{l} x_{i} \leq \sum_{i=1}^{l} y_{i} \quad \text { for } \quad 1 \leq l<d,
\end{gathered}
$$

and

$$
\begin{aligned}
x_{1} x_{d} & =y_{1}\left(y_{d}+\epsilon+\delta\right) \\
& >y_{1} y_{d} \geq y_{t+1}^{2} \\
& >\left(y_{t+1}-\frac{\epsilon}{\triangle}\right)^{2}=x_{t+1}^{2},
\end{aligned}
$$

so we have $x \notin M(y)$ from Lemma 2. That completes our proof.

Suppose $t$ and $m$ denote the numbers of the components which are equal to $y_{n}$ and which are equal to $y_{1}$, respectively. Let $d$ be the maximal index of the components which are greater than $y_{n-t}$. If $d>m+1$ and $y_{n} y_{d} \leq y_{n-t}^{2}$ then we can also deduce that $T(y) \nsubseteq M(y)$.

An interesting special case of Theorem 3 is when $n>$ 4 , if $y_{1}>y_{2}>y_{3}>y_{n-1}>y_{n}$ and $y_{1} y_{3} \geq y_{2}^{2}$ then $T(y) \nsubseteq M(y)$.

Theorem 3 in fact gives us a method to construct a vector $y$ for which $T(y) \nsubseteq M(y)$. To be more specific, given a 
vector $\bar{y}$ such that $T(\bar{y}) \neq S(\bar{y})$, we can derive a desired $y$ by the following two steps. First, add a sufficiently large component to $\bar{y}$ such that the conditions presented in Theorem 3 are satisfied for the new vector (notice that from Theorem 6 of $[9]$, when $T(\bar{y}) \neq S(\bar{y})$, the condition $d<n-m$ in Theorem 3 holds automatically); second, normalize the vector to $y$ such that it is a probability vector. For example, given $\bar{y}=(0.5,0.25,0.25,0)$, we can derive $y=(3,0.5,0.25,0.25,0) / 4$ and $T(y) \nsubseteq M(y)$. Furthermore, since the proof of Theorem 3 is constructive, the states which can be transformed into $y$ by catalystassisted transformation while cannot by multiple-copy transformation can also be constructed.

We have proved that $T(y) \neq M(y)$ in some cases. Moreover, witness vectors which are in $T(y)$ but not in $M(y)$ are also constructed explicitly. It should be pointed out, however, that the witness vectors we constructed lie on the boundary of $T(y)$ without any exception, that is, they all satisfy the property that $x_{1}^{\downarrow}=y_{1}^{\downarrow}$ or $x_{n}^{\downarrow}=y_{n}^{\downarrow}$. These witness vectors can be involved if we consider the closure of $M(y)$ instead. In fact, we will see in the following section that in probabilistic setting, the two sets $M^{\lambda}(y)$ and $T^{\lambda}(y)$ defined in Eqs.(26) and (27) have exactly the same closure for $0 \leq \lambda<1$. So the question remained is to show whether or not $M(y)$ and $T(y)$ also have the same closure.

\section{PROBABILISTIC CASE}

We considered deterministic entanglement transformations in the previous section. In this section, let us turn to examine transformations with maximal probability strictly less than 1 .

Given a nonnegative number $\lambda<1$, let

$$
S^{\lambda}(y)=\left\{x \in V^{n} \mid P(x \rightarrow y) \geq \lambda\right\}
$$

be the set of states that can be transformed into $y$ by LOCC with the maximal probability not less than $\lambda$,

$$
T^{\lambda}(y)=\left\{x \in V^{n} \mid \exists c, P(x \otimes c \rightarrow y \otimes c) \geq \lambda\right\}
$$

be the set of states that can be transformed into $y$ by catalyst-assisted LOCC with the maximal probability not less than $\lambda$, and

$$
M^{\lambda}(y)=\left\{x \in V^{n} \mid \exists k, P\left(x^{\otimes k} \rightarrow y^{\otimes k}\right)^{1 / k} \geq \lambda\right\}
$$

the set of states which, when some appropriate number of copies are provided, can be transformed into the same number of $y$ by multiple-copy LOCC with the maximal geometric average probability not less than $\lambda$. We have proved in [13] that $M^{\lambda}(y) \subseteq T^{\lambda}(y)$. In the following we further show that the reverse is not always true. For this purpose, two lemmas which corresponding to Lemma 1 and Lemma 2 in the previous section are useful.

Lemma 4 Suppose $x, y \in V^{n}$ and $z \in V^{m}$ are nonincreasingly arranged nonnegative vectors. If $x \in T^{\lambda}(y)$ then $x^{\prime} \oplus \lambda z \in T^{\lambda}(y \oplus z)$, where $x^{\prime}=\left(x_{1}^{\prime}, x_{2}, \ldots, x_{n}\right)$ with $x_{1}^{\prime}=x_{1}+(1-\lambda) \sum_{i=1}^{m} z_{i}$.

Proof. From $x \in T^{\lambda}(y)$, there exists $c \in V^{r}$ such that $P(x \otimes c \rightarrow y \otimes c) \geq \lambda$. For any arbitrarily $1 \leq l \leq$ $(n+m) r$, we have

$$
\sum_{i=l}^{(n+m) r}\left(\left(x^{\prime} \oplus \lambda z\right) \otimes c\right)_{i}^{\downarrow}=\sum_{i=l_{1}}^{n r}\left(x^{\prime} \otimes c\right)_{i}^{\downarrow}+\lambda \sum_{i=l_{2}}^{m r}(z \otimes c)_{i}^{\downarrow}
$$

for some $1 \leq l_{1} \leq n r$ and $1 \leq l_{2} \leq m r$. On the other hand, by definition

$$
\sum_{i=l}^{(n+m) r}((y \oplus z) \otimes c)_{i}^{\downarrow} \leq \sum_{i=l_{1}}^{n r}(y \otimes c)_{i}^{\downarrow}+\sum_{i=l_{2}}^{m r}(z \otimes c)_{i}^{\downarrow}
$$

Notice that $x_{i}^{\prime} \geq x_{i}$ for any $i=1, \ldots, n$, and that from $P(x \otimes c \rightarrow y \otimes c) \geq \lambda$ we know

$$
\sum_{i=l_{1}}^{n r}(x \otimes c)_{i}^{\downarrow} \geq \lambda \sum_{i=l_{1}}^{n r}(y \otimes c)_{i}^{\downarrow} .
$$

It follows from Eqs. (28)- that

$$
\sum_{i=l}^{(n+m) r}\left(\left(x^{\prime} \oplus \lambda z\right) \otimes c\right)_{i}^{\downarrow} \geq \lambda \sum_{i=l}^{(n+m) r}((y \oplus z) \otimes c)_{i}^{\downarrow}
$$

and $P\left(\left(x^{\prime} \oplus \lambda z\right) \otimes c \rightarrow(y \oplus z) \otimes c\right) \geq \lambda$ from the arbitrariness of $l$. So we have $x^{\prime} \oplus \lambda z \in T^{\bar{\lambda}}(y \oplus z)$.

Lemma 5 Suppose $x$ and $y$ are two nonincreasingly arranged $n$-dimensional probability vectors, $x_{n}=\lambda y_{n}$ but $x \notin S^{\lambda}(y)$ for $\lambda \in(0,1)$. Let

$$
d=\max \left\{l: 1 \leq l \leq n, \sum_{i=l}^{n} x_{i}<\lambda \sum_{i=l}^{n} y_{i}\right\}
$$

Denote by $t_{1}$ the number of components in $x$ which are equal to $x_{n}$, while $t_{2}$ the number of components in $y$ which are equal to $y_{n}$. If $t_{1}=t_{2}=t$ and

$$
x_{n} x_{d} \leq x_{n-t}^{2} \quad \text { and } \quad y_{n} y_{d} \leq y_{n-t}^{2},
$$

then $x \notin M^{\lambda}(y)$.

Proof. Similar to Lemma 2. But the last $t^{k}+k t(n-t-d)$ components of $\left(x^{\otimes k}\right)^{\downarrow}$ and $\left(y^{\otimes k}\right)^{\downarrow}$ are considered for any $k$ at this time.

Theorem 6 Let $\lambda \in(0,1)$. There exists $y \in V^{n}$ such that $T^{\lambda}(y) \nsubseteq M^{\lambda}(y)$.

Proof. The proof is similar to but more complicated than that of Theorem 3. Beside, due to the asymmetry of roles of the largest and the smallest components in determining the maximal transforming probability presented in Eq.(2), components at the tail but not at the head of $y$ 
should be examined. We outline the main steps of the proof here.

Let $y \in V^{n}$ such that

$$
y_{1}=\cdots=y_{m}>y_{m+1},
$$

and

$$
y_{d}>y_{d+1}=\cdots=y_{n-t}>y_{n-t+1}=\cdots=y_{n} .
$$

where $d>m+1$ and $y_{n} y_{d} \leq y_{n-t}^{2}$. Take a positive number $\epsilon$ such that

$$
\epsilon<\min \left\{\frac{\lambda m}{m+1}\left(y_{m}-y_{m+1}\right), \frac{\lambda \triangle}{\triangle+1}\left(y_{d}-y_{d+1}\right)\right\},
$$

where $\triangle=n-t-d$. Define

$$
\begin{gathered}
\bar{x}=\left(\widetilde{y_{1}}, \lambda y_{2}-\frac{\epsilon}{m}, \cdots, \lambda y_{m}-\frac{\epsilon}{m}, \lambda y_{m+1}+\epsilon, \lambda y_{m+2},\right. \\
\left.\cdots, \lambda y_{d-1}, \lambda y_{d}-\epsilon, \lambda y_{d+1}+\frac{\epsilon}{\triangle}, \cdots, \lambda y_{n-t}+\frac{\epsilon}{\triangle}\right)
\end{gathered}
$$

and

$$
\bar{y}=\left(y_{1}, y_{2}, \cdots, y_{n-t}\right) .
$$

Here $\widetilde{y_{1}}=y_{1}+(1-\lambda) \sum_{i=2}^{n-t} y_{i}-\epsilon / m$. Then $\bar{x}$ and $\bar{y}$ are both nonincreasingly arranged, and $\bar{x} \in S^{\lambda}(\bar{y})$. Furthermore, $\bar{x}$ is in the interior of $T^{\lambda}(\bar{y})$ by Theorem 9 in 10$]$ since $\bar{x}_{n-t}>\lambda \bar{y}_{n-t}$. So there exists a sufficiently small but positive $\delta$ such that $\bar{x}^{\prime} \in T^{\lambda}(\bar{y})$ where

$$
\begin{aligned}
\bar{x}^{\prime \downarrow}= & \left(\widetilde{y_{1}}, \lambda y_{2}-\frac{\epsilon}{m}, \cdots, \lambda y_{m}-\frac{\epsilon}{m}, \lambda y_{m+1}+\epsilon+\delta,\right. \\
& \lambda y_{m+2}, \cdots, \lambda y_{d-1}, \lambda y_{d}-\epsilon-\delta, \lambda y_{d+1}+\frac{\epsilon}{\triangle} \\
& \left.\cdots, \lambda y_{n-t}+\frac{\epsilon}{\triangle}\right) .
\end{aligned}
$$

Now define $x=\left(x^{\prime}, \lambda y_{n-t+1}, \cdots, \lambda y_{n}\right)$, where $x^{\prime}=$ $\left(x_{1}^{\prime}, \bar{x}_{2}^{\prime \downarrow}, \ldots, \bar{x}_{n-t}^{\prime \downarrow}\right)$ and $x_{1}^{\prime}=y_{1}+(1-\lambda) \sum_{i=2}^{n} y_{i}-\epsilon / m$. By Lemma 4 and 5 we can similarly prove that $x \in T^{\lambda}(y)$ but $x \notin M^{\lambda}(y)$.

To draw a clearer picture of the relation between catalyst-assisted transformation and multiple-copy transformation in purely probabilistic setting, we investigate the limit properties of $T^{\lambda}(y)$ and $M^{\lambda}(y)$ about $\lambda$. Since $T^{\lambda^{\prime}}(y) \subseteq T^{\lambda}(y)$ for any $\lambda^{\prime}>\lambda$, we can define

$$
T^{\lambda-}(y)=\bigcap_{\lambda^{\prime}<\lambda} T^{\lambda^{\prime}}(y), \quad T^{\lambda+}(y)=\bigcup_{\lambda^{\prime}>\lambda} T^{\lambda^{\prime}}(y)
$$

which denote respectively the left limit and right limit of the set-valued function $T^{\lambda}(y)$ at the point $\lambda$. Similar notions can be defined for $M^{\lambda+}(y)$ and $M^{\lambda-}(y)$. It is direct from the definition that

$$
\begin{aligned}
T^{\lambda-}(y) & =\left\{x \mid \sup _{c} P(x \otimes c \rightarrow y \otimes c) \geq \lambda\right\}, \\
M^{\lambda-}(y) & =\left\{x \mid \sup _{k} P\left(x^{\otimes k} \rightarrow y^{\otimes k}\right)^{1 / k} \geq \lambda\right\},
\end{aligned}
$$

and we have shown in [13] that $T^{\lambda-}(y)=M^{\lambda-}(y)$ for any $\lambda \in[0,1]$.

The following theorem tells us that generally, the function $T^{\lambda}(y)$ is neither left continuous nor right continuous at any point $\lambda \in(0,1)$, although it is 'almost' right continuous in the sense that the right limit at $\lambda$ shares the same interior points with $T^{\lambda}(y)$.

Theorem 7 For any $y \in V^{n}$ and $0<\lambda<1$,

1) $T^{\lambda+}(y)$ is open while $T^{\lambda-}(y)$ is closed,

2) $T^{\lambda+}(y) \varsubsetneqq T^{\lambda}(y) \subseteq T^{\lambda-}(y)$, and when $y_{n}^{\downarrow}>0$, $T^{\lambda}(y)=T^{\lambda-}(y)$ if and only if $y_{2}^{\downarrow}=y_{n}^{\downarrow}$,

3) $T^{\lambda}(y)^{\circ}=T^{\lambda+}(y)$.

Proof. 1). For any $x \in T^{\lambda+}(y)$, there exist $\mu>\lambda$ and $c$ such that $P(x \otimes c \rightarrow y \otimes c) \geq \mu$. Let $\nu$ be a number such that $\mu>\nu>\lambda$. From the continuity of $P(x \otimes c \rightarrow y \otimes c)$ about $x$ for fixed $c$ and $y$, we have some $\epsilon>0$, such that for any $x^{\prime} \in B(x, \epsilon)$,

$$
\left|P\left(x^{\prime} \otimes c \rightarrow y \otimes c\right)-P(x \otimes c \rightarrow y \otimes c)\right|<\mu-\nu .
$$

We then derive that $x^{\prime} \in T^{\lambda+}(y)$ since

$$
\begin{aligned}
P\left(x^{\prime} \otimes c \rightarrow y \otimes c\right) & \geq P(x \otimes c \rightarrow y \otimes c)-A \\
& >\mu-(\mu-\nu)=\nu
\end{aligned}
$$

where $A=\left|P\left(x^{\prime} \otimes c \rightarrow y \otimes c\right)-P(x \otimes c \rightarrow y \otimes c)\right|$. That completes the proof that $T^{\lambda+}(y)$ is open. To prove the closeness of $T^{\lambda-}(y)$, we take any sequence $x_{i} \in T^{\lambda-}(y)$ such that $\lim _{i} x_{i}=x$. By definition, for any $x_{i}$ we have $\sup _{c} P\left(x_{i} \otimes c \rightarrow y \otimes c\right) \geq \lambda$. To realize the transformation from $x \otimes c$ to $y \otimes c$, a possible protocol is first transforming $x$ to $x_{i}$ and then transforming $x_{i} \otimes c$ to $y \otimes c$. So we have the following relation

$$
P(x \otimes c \rightarrow y \otimes c) \geq P\left(x \rightarrow x_{i}\right) P\left(x_{i} \otimes c \rightarrow y \otimes c\right) .
$$

Thus

$$
\begin{aligned}
& \sup _{c} P(x \otimes c \rightarrow y \otimes c) \\
& \geq P\left(x \rightarrow x_{i}\right) \sup _{c} P\left(x_{i} \otimes c \rightarrow y \otimes c\right) \\
& \geq \lambda P\left(x \rightarrow x_{i}\right) .
\end{aligned}
$$

The desired result that $x \in T^{\lambda-}(y)$ follows from the above equation by letting $i$ tend to infinity.

2 ) is obvious from 1) and the fact that $T^{\lambda}(y)$ is neither closed nor open when $y_{2}^{\downarrow}>y_{n}^{\downarrow}$ (see the first two lines of the proof of Theorem 11 of [10]). When $y_{2}^{\downarrow}=y_{n}^{\downarrow}$, we have $T^{\lambda}(y)=S^{\lambda}(y)$ (Theorem 10 of 10$]$ ), then $T^{\lambda}(y)=$ $T^{\lambda-}(y)$ follows from the continuity of $S^{\lambda}(y)$ for any $0<$ $\lambda<1$ (Theorem 3 of [10]).

Now we prove 3$)$. The relation $T^{\lambda+}(y) \subseteq T^{\lambda}(y)^{\circ}$ is easy from 1) and 2). To prove the reverse relation, we take any $x \in T^{\lambda}(y)^{\circ}$. Then $P(x \otimes c \rightarrow y \otimes c) \geq \lambda$ for some $c$. There are two cases to consider. 
Case 1. $P(x \otimes c \rightarrow y \otimes c)>\lambda$. In this case, we know immediately that $x \in T^{\lambda^{\prime}}(y) \subseteq T^{\lambda+}(y)$ for $\lambda^{\prime}=$ $P(x \otimes c \rightarrow y \otimes c)$.

Case 2. $P(x \otimes c \rightarrow y \otimes c)=\lambda$. Since $x$ is an interior point of $T^{\lambda}(y)$, from Theorem 9 of [10] we have $x_{n}^{\downarrow} / y_{n}^{\downarrow}>\lambda$. Then $P(x \otimes c \rightarrow y \otimes c)<\min \left\{1, x_{n}^{\downarrow} / y_{n}^{\downarrow}\right\}$. By Theorem 2 of [14], there exists a catalyst $c^{\prime}$ such that

$$
P\left(x \otimes c \otimes c^{\prime} \rightarrow y \otimes c \otimes c^{\prime}\right)>P(x \otimes c \rightarrow y \otimes c)=\lambda .
$$

So we also have $x \in T^{\lambda^{\prime}}(y) \subseteq T^{\lambda+}(y)$ for $\lambda^{\prime}=P(x \otimes c \otimes$ $\left.c^{\prime} \rightarrow y \otimes c \otimes c^{\prime}\right)$.

Notice that we assume $y_{n}^{\downarrow}>0$ in 2) of the above theorem. When $y_{n}^{\downarrow}=0$, it is not clear till now whether or not the result still holds.

With similar techniques, we can prove a corresponding result of Theorem $\mathbf{7}$ for probabilistic multiple-copy transformation.

Theorem 8 For any $y \in V^{n}$ and $0<\lambda<1$,

1) $M^{\lambda+}(y)$ is open while $M^{\lambda-}(y)$ is closed,

2) $M^{\lambda+}(y) \varsubsetneqq M^{\lambda}(y) \subseteq M^{\lambda-}(y)$, and when $y_{n}^{\downarrow}>0$, $M^{\lambda}(y)=M^{\lambda-}(y)$ if and only if $y_{2}^{\downarrow}=y_{n}^{\downarrow}$,

3) $M^{\lambda}(y)^{\circ}=M^{\lambda+}(y)$.

Proof. Similar to the proof of Theorem 7.

Now we can show our main result of this section. Rather surprisingly, when the probability threshold $\lambda$ is strictly less than 1 , probabilistic catalyst-assisted transformation and probabilistic multiple-copy transformation are geometrically equivalent in the sense that the two sets $T^{\lambda}(y)$ and $M^{\lambda}(y)$ in fact share the same interior points (or equivalently, the same closure).

Lemma 9 If $0 \leq \lambda<\lambda^{\prime} \leq 1$, then $T^{\lambda^{\prime}}(y) \subseteq M^{\lambda}(y)$.

Proof. By definition, for any $x \in T^{\lambda^{\prime}}(y)$, there exists $c$ such that $P(x \otimes c \rightarrow y \otimes c) \geq \lambda^{\prime}$. Then from Theorem 1 of [13], we have

$$
\sup _{k} P\left(x^{\otimes k} \rightarrow y^{\otimes k}\right)^{1 / k}=\sup _{c} P(x \otimes c \rightarrow y \otimes c) \geq \lambda^{\prime}>\lambda .
$$

Thus there exists $k_{0}$ such that $P\left(x^{\otimes k_{0}} \rightarrow y^{\otimes k_{0}}\right)^{1 / k_{0}}>\lambda$. So $x \in M^{\lambda}(y)$.

Theorem 10 For any $y \in V^{n}$ and $0 \leq \lambda<1$, we have $\overline{T^{\lambda}(y)}=\overline{M^{\lambda}(y)}$.

Proof. From Theorems 7 and 8 to prove this theorem we need only show that $T^{\lambda+}(y)=M^{\lambda+}(y)$, or $T^{\lambda+}(y) \subseteq$ $M^{\lambda+}(y)$ since the reverse is obvious. For any $\lambda^{\prime}>\lambda$, take $\mu$ such that $\lambda^{\prime}>\mu>\lambda$. Then from Lemma 9 we have $T^{\lambda^{\prime}}(y) \subseteq M^{\mu}(y) \subseteq M^{\lambda+}(y)$. So $T^{\lambda+}(y) \subseteq M^{\lambda+}(y)$ by definition.

We are now in the appropriate position to elaborate the difference between the geometrical equivalence shown in this paper and the asymptotical equivalence proven in [13. The latter can be expressed with our notations as $T^{\lambda-}(y)=M^{\lambda-}(y)$ while our result here indicates that $\overline{T^{\lambda}(y)}=\overline{M^{\lambda}(y)}$. Since the question whether or not $T^{\lambda-}(y)=\overline{T^{\lambda}(y)}$ (or equivalently, $M^{\lambda-}(y)=\overline{M^{\lambda}(y)}$ ) remains open, our result cannot be derived directly from the one in [13].

\section{CONCLUSION}

To summarize, we show that in some cases catalystassisted entanglement transformation is strictly more powerful than multiple-copy one in either deterministic or probabilistic setting. For purely probabilistic setting, however, we can prove that these two kinds of transformations are geometrically equivalent in the sense that the two sets $T^{\lambda}(y)$ and $M^{\lambda}(y)$, denoting the sets of bipartite pure states which can be converted into a given state with Schmidt coefficient vector $y$ with maximal probabilities not less than $\lambda$ by catalyst-assisted transformation and by multiple-copy transformation, respectively, have the same closure. The limit properties of $T^{\lambda}(y)$ and $M^{\lambda}(y)$ as set-valued functions about $\lambda$ are also discussed.

The results about the relation between catalystassisted transformation and multiple-copy transformation shown in this paper and our previous works can be described by the following diagrams:

$$
\begin{aligned}
& M^{\lambda+}(y)=M^{\lambda}(y)^{\circ} \varsubsetneqq M^{\lambda}(y) \varsubsetneqq \overline{M^{\lambda}(y)} \subsetneq M^{\lambda-}(y) \\
& \|\quad\| \quad \wedge \quad \| \\
& T^{\lambda+}(y)=T^{\lambda}(y)^{\circ} \varsubsetneqq T^{\lambda}(y) \varsubsetneqq \overline{T^{\lambda}(y)} \subseteq T^{\lambda-}(y)
\end{aligned}
$$

for purely probabilistic case $(\lambda<1)$ and

$$
\begin{array}{ccccc}
M(y)^{\circ} & \varsubsetneqq M(y) & \varsubsetneqq \overline{M(y)} & \subsetneq M^{-}(y) \\
\cap \downarrow & \curvearrowright & & \cap \downarrow & \| \\
T(y)^{\circ} & \varsubsetneqq T(y) & \varsubsetneqq \overline{T(y)} & \subsetneq T^{-}(y)
\end{array}
$$

for deterministic case. Where we write $A(y) \varsubsetneqq B(y)$ if $A(y) \subseteq B(y)$ holds for all $y$ but there exists some $y$ such that $A(y) \neq B(y)$; while by $A(y) \subset B(y)$ we indicate that whether or not there exists $y$ such that $A(y) \neq B(y)$ is still open, although $A(y) \subseteq B(y)$ always holds for all $y$.

From the above two diagrams, the remaining questions for further study are:

1). Whether or not $\overline{T^{\lambda}(y)}=T^{\lambda-}(y)$ (or equivalently, $\left.\overline{M^{\lambda}(y)}=M^{\lambda-}(y)\right)$ for any $y$ and $\lambda \leq 1$. In other words, whether or not the function $T^{\lambda}(y)$ (or $\left.M^{\lambda}(y)\right)$ is 'almost' left continuous at any $\lambda \leq 1$.

2). Whether or not $\overline{\overline{T(y)}}=\overline{M(y)}$ (or equivalently, $\left.T(y)^{\circ}=M(y)^{\circ}\right)$ for any $y$. That is, whether or not catalyst-assisted transformation and multiple-copy transformation are also geometrically equivalent in deterministic setting. 


\section{ACKNOWLEDGMENTS}

The authors thank the colleagues in the Quantum Computation and Quantum Information Research Group for useful discussion. This work was partly supported by the Natural Science Foundation of China (Grant Nos. 60503001, 60321002, and 60305005), and by Tsinghua Basic Research Foundation (Grant No. 052220204). R. Duan acknowledges the financial support of Tsinghua University (Grant No. 052420003).
[1] C. H. Bennett and G. Brassard, in Proceedings of IEEE International Conference on Computers, Systems and Signal Processing, Bangalore, India, pp. 175-179 (1984).

[2] C. H. Bennett and S. J. Wiesner, Physical Review Letters 69, 2881 (1992), URL http://link.aps.org/abstract/PRL/v69/p2881

[3] C. H. Bennett, G. Brassard, C. Crepeau, R. Jozsa, A. Peres, and W. K. Wootters, Physical Review Letters 70, 1895 (1993), URL http://link.aps.org/abstract/PRL/v70/p1895.

[4] C. H. Bennett, H. J. Bernstein, S. Popescu, and B. Schumacher, Physical Review A (Atomic, Molecular, and Optical Physics) 53, 2046 (1996), URL http://link.aps.org/abstract/PRA/v53/p2046

[5] M. A. Nielsen, Physical Review Letters 83, 436 (1999), URL http://link . aps . org/abstract/PRL/v83/p436

[6] A. W. Marshall and I. Olkin, Inequalities: Theory of Majorization and Its Applications, Academic Press, New York (1979).

[7] G. Vidal, Physical Review Letters 83, 1046 (1999), URL http://link.aps.org/abstract/PRL/v83/p1046

[8] D. Jonathan and M. B. Plenio, Physical Review Letters 83, 3566 (1999), URL http://link.aps.org/abstract/PRL/v83/p3566
[9] S. Daftuar and M. Klimesh, Physical Review A (Atomic, Molecular, and Optical Physics) 64, 042314 (pages 6) (2001), URL http://link.aps.org/abstract/PRA/v64/e042314

[10] Y. Feng, R. Duan, and M. Ying, IEEE Transactions on Information Theory 51, 1090 (2005).

[11] S. Bandyopadhyay, V. Roychowdhury, and U. Sen, Physical Review A (Atomic, Molecular, and Optical Physics) 65, 052315 (pages 4) (2002), URL http://link.aps.org/abstract/PRA/v65/e052315

[12] R. Duan, Y. Feng, X. Li, and M. Ying, Physical Review A (Atomic, Molecular, and Optical Physics) 71, 042319 (pages 10) (2005), URL http://link.aps.org/abstract/PRA/v71/e042319

[13] R. Duan, Y. Feng, and M. Ying, Physical Review A (Atomic, Molecular, and Optical Physics) 72, 024306 (pages 4) (2005), URL http://link.aps.org/abstract/PRA/v72/e024306

[14] Y. Feng, R. Duan, and M. Ying, Physical Review A (Atomic, Molecular, and Optical Physics) 69, $062310 \quad$ (pages 5) (2004), URL http://link.aps.org/abstract/PRA/v69/e062310 